\title{
Dynamics of fixation of advantageous mutations
}

\author{
Viviane M. de Oliveirałand Paulo R. A. Campos $\ddagger$ \\ October 30, 2018
}

\begin{abstract}
Instituto de Física Gleb Wataghin, Universidade Estadual de Campinas 13083-970 Campinas SP, Brazil

Corresponding author: Paulo R. A. Campos. Phone: 55-19-3788-5373, Fax: 55-19-3788-5376, email: prac@ifi.unicamp.br
\end{abstract}

Keywords: fixation, branching processes, population genetics 


\begin{abstract}
We investigate the process of fixation of advantageous mutations in an asexual population. We assume that the effect of each beneficial mutation is exponentially distributed with mean value $\omega_{\text {med }}=1 / \beta$. The model also considers that the effect of each new deleterious mutation reduces the fitness of the organism independent on the previous number of mutations. We use the branching process formulation and also extensive simulations to study the model. The agreement between the analytical predictions and the simulational data is quite satisfactory. Surprisingly, we observe that the dependence of the probability of fixation $P_{f i x}$ on the parameter $\omega_{m e d}$ is precisely described by a power-law relation, $P_{f i x} \sim \omega_{\text {med }}^{\gamma}$. The exponent $\gamma$ is an increase function of the rate of deleterious mutations $U$, whereas the probability $P_{f i x}$ is a decreasing function of $U$. The mean value $\omega_{f i x}$ of the beneficial mutations which reach ultimate fixation depends on $U$ and $\omega_{\text {med }}$. The ratio $\omega_{\text {fix }} / \omega_{\text {med }}$ increases as we consider higher values of mutation value $U$ in the region of intermediate to large values of $\omega_{\text {med }}$, whereas for low $\omega_{m e d}$ we observe the opposite behavior.
\end{abstract}

\title{
1 Introduction
}

The process of adaptation in evolving populations takes place by the continuous production of beneficial mutations and the ultimate fixation of these variants in the population. This mechanism is relevant not only to improve the adaptation of the organisms to the environment, but also to prevent that in an environment where a large supply of slightly deleterious mutations persists, the population presents a continuous decline of the mean population fitness leading to the extinction of the whole population. Besides, beneficial mutations have a crucial role to permit the adaptation in dynamic environments where drastic changes take place. The attempt to understand the dynamics of fixation of beneficial mutations stems from the classical population genetics with the pioneer works of Fisher and Haldane (Fisher 1922; Fisher 1930. Haldane 1927). With the developments in the experimental biology and the consequent abundance of data from real biological systems, especially those from bacteria (de Visser et al. 1999, Rozen et al. 2002, Shaver et al. 2002) and viruses populations (Miralles et al. 1999; Cuevas et al. 2002), it is now possible to better understand the main mechanisms underlying the process of adaptation in these populations and the rhythm at which it oc- 
curs. In this sense, recent theoretical developments have contributed to the advances in the field (Gerrish and Lenski 1998, Orr 2000, Barton 1995, Johnson and Barton 2002, Gerrish 2001). For instance, it is known that the increase of the supply of beneficial mutations in an asexual population does not result in a linear response of the evolutionary process due to the competition between distinct lineages in order to reach fixation. In an asexual population beneficial mutations are fixed sequentially and in the case where two or more mutations compete for fixation only one mutation can be kept in the population, with the definitive loss of the remaining ones. This process is named clonal interference(Hill and Robertson 1966, Campos et al. 2003).

Here, we consider that beneficial mutations are rare events and so they do not compete for fixation with other mutations. Nevertheless, deleterious mutations occur at a constant rate $U$. Deleterious mutations affect drastically the dynamics of fixation of the beneficial mutations. As previously demonstrated (Campos 2003, Peck 1994), the probability of fixation $P_{f i x}$ of advantageous mutants is a decrease function of the mutation rate $U$. This is a consequence of the possibility of occurrence of such beneficial mutation in a genome with a large amount of segregated deleterious mutations, i.e., in a genome with very low fitness value. In this paper we consider the multiplicative fitness landscape at which the effect of each new deleterious mutation is independent of other genes.

The paper is organized in the following way: In the next Section we describe the model. In Section III we discuss the branching process formulation. In Section IV we show our results, and finally In Section $\mathrm{V}$ we present our conclusions.

\section{The evolutionary model}

The population consists of $N$ asexual haploid organisms that evolve according to the Wright-Fisher model. In the model, the individuals in generation $t+1$ are direct descendants of the individuals in time $t$. The probability that an organism is descendant of a particular parent in the previous generation is proportional to the parent's fitness. We also assume that the genome of an individual is represented by an infinitely large sequence of bits $\mathbf{S}=$ $\left(s_{1}, s_{2}, \ldots, s_{\infty}\right)$, where the digit $s_{\alpha}$ denotes the state of gene $\alpha$, which can take two distinct values $s_{\alpha}=0,1$. The state $s_{\alpha}=0$ means that the digit $\alpha$ remains in the original state of the ancestor of the population, whereas the 


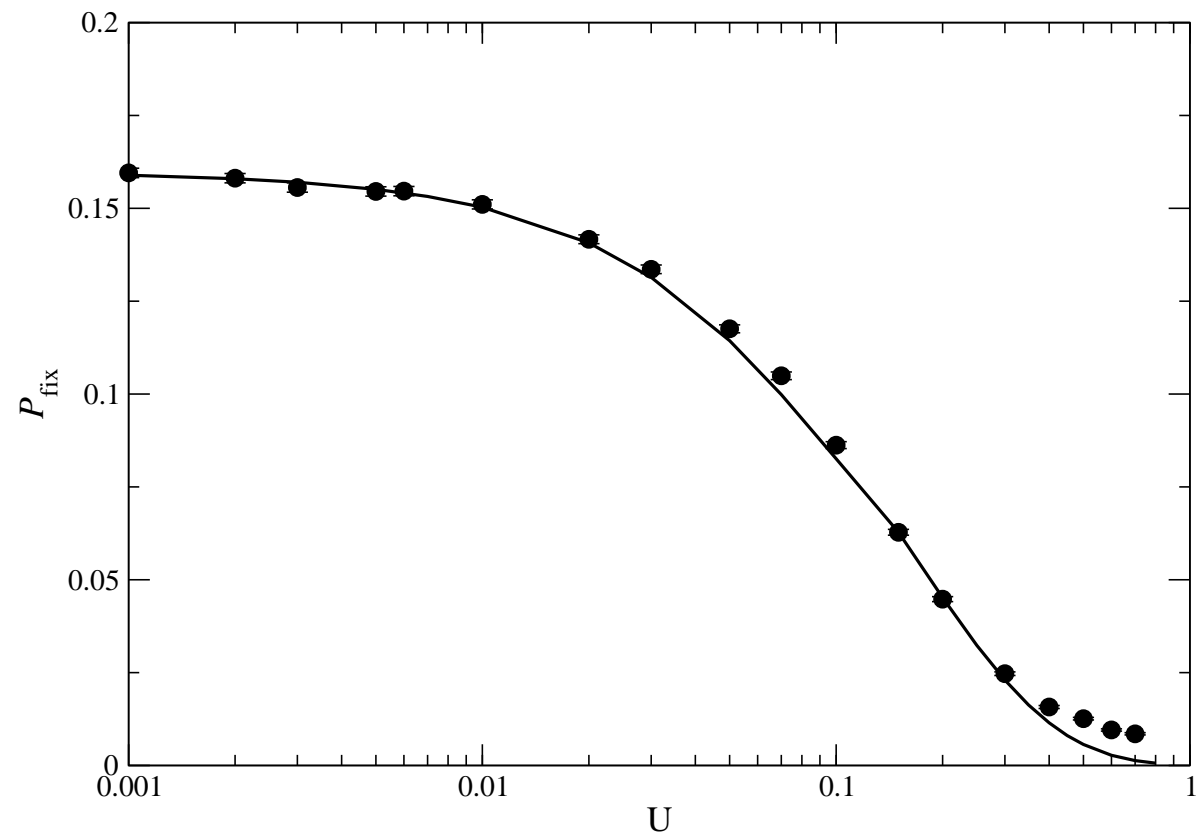

Figure 1: Probability of ultimate fixation as a function of the mutation rate $U$. The parameters are $N=1000, \beta=10$ and $s_{d}=0.1$. The data points correspond to the simulation results over 100,000 runs, and the thick line is the theoretical prediction according to Eq. (11).

state $s_{\alpha}=1$ means that the nucleotide $\alpha$ has been hit by a mutation. Because we assume infinitely large genomes, the probability of reverse mutations is negligible, i.e., we consider that only transitions of the type $s_{\alpha}=0 \rightarrow 1$ can take place.

When a newborn individual arises it acquires the number of mutations present in its parent's genome and an additional amount of new deleterious mutations $n$ taken from a Poisson distribution with parameter $U$, where $U$ is the mean number of new mutations per individual per generation. The aforesaid model was introduced by Kimura and Watterson (Kimura and Crow 1964; Watterson 1975) and it is referred to the infinitesites model. The fitness of an individual depends on the total number $k$ of mutations in its genome and is given by

$$
w_{k}=\left(1-s_{d}\right)^{k}
$$

where $s_{d}$ is the cost associated to each deleterious mutation. This case cor- 
responds to the multiplicative landscape, where each new mutation reduces the fitness of the organism by the same factor (Campos et al. 2000).

For infinitely large populations, the distribution of frequencies of the class of individuals with $k$ mutations in the equilibrium regime, which we denote by $\bar{C}_{k}$, can be calculated by the following set of equations

$$
\bar{C}_{k}=\frac{1}{w_{m}-w_{k}} \sum_{j=m}^{k-1} \frac{U^{k-j}}{(k-j) !} w_{j} \bar{C}_{j} \quad k>m .
$$

Using the above expression we can recursively calculate the ratios $\bar{C}_{k} / \bar{C}_{m}$ and estimate $\bar{C}_{m}$ from the normalization condition $\sum_{K} \bar{C}_{k}=1$ (Colato and Fontanari 2001), where $m$ is the index of the class of the fittest individuals existing in the population. In our analysis we always assume $m=0$.

In our simulations, the initial population is distributed in different classes of individuals according to the frequencies of equilibrium, given by Eq. (2). In this case, it is not necessary to wait the population evolves up to reaching the stationary regime.

In the first generation, we randomly select an individual which acquires an advantageous mutation with selective effect $s_{b}$ obtained from an exponential distribution

$$
g\left(s_{b}\right)=\beta \exp \left(-\beta s_{b}\right),
$$

which is the expected distribution, as argued by the extreme value theory (Gillespie 1991; Orr 2003). Whether this mutation happens in a genotype with $k$ deleterious mutations its adaptation value increases by a factor $\left(1+s_{b}\right)$, i.e.,

$$
w_{k}=\left(1+s_{b}\right)\left(1-s_{d}\right)^{k} .
$$

The advantageous mutation can be propagated for future generations as soon as the individuals which have acquired it replicate. The fitness of those individuals carrying the beneficial mutation takes the same form as in Eq. (4.)

In our approach, we consider the beneficial mutation to be fixed when the genotype that has first acquired it becomes the most-recent common ancestor of the whole population (Barton 1995, Johnson and Barton 2002, Campos 2003, Wilke 2003, Campos et al. 2003). 


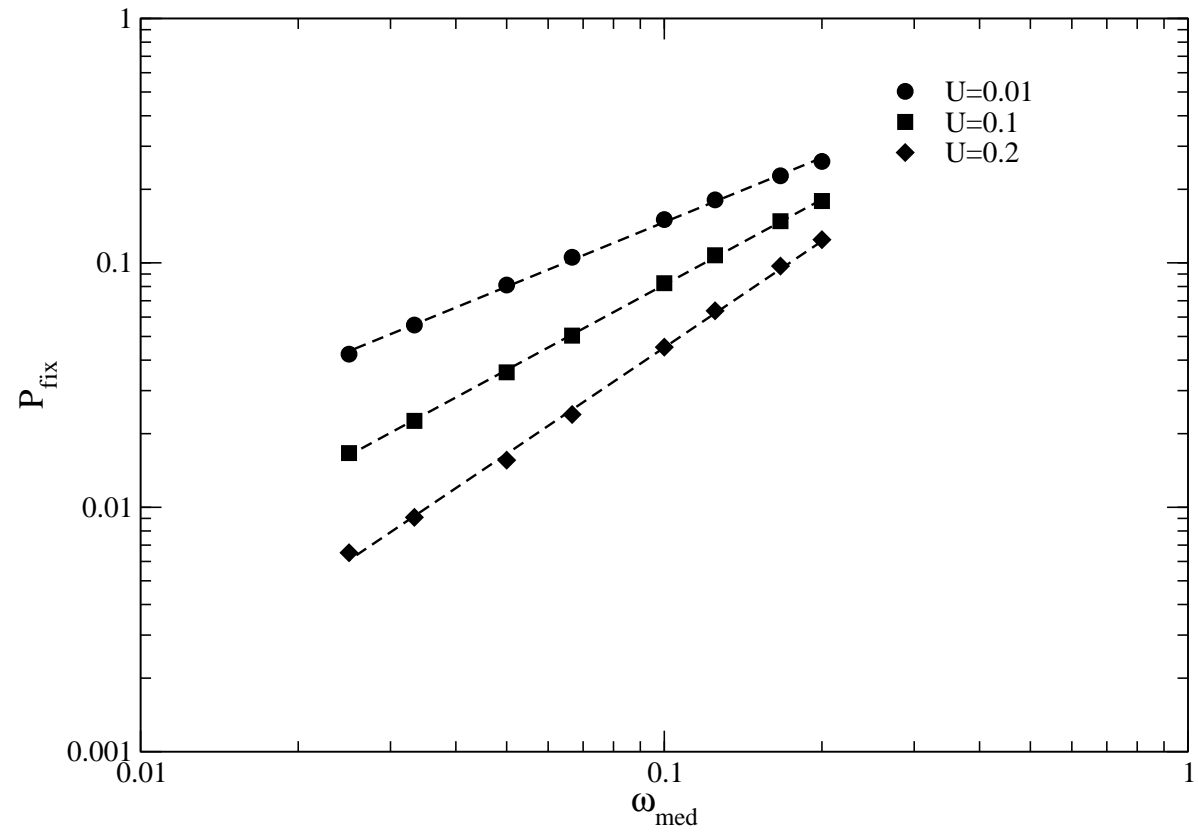

Figure 2: Probability of fixation $P_{f i x}$ as a function of the parameter $\omega_{\text {med }}$ for fixed values of mutation rate $U$. The data points are the theoretical predictions and the dashed-lines are the best fits which give a power-law distribution. The parameter values are $s_{d}=0.1$ and from top to bottom $U=0.01, U=0.1$ and $U=0.2$.

\section{Branching Process Formulation}

The theory of branching process (Harris 1963) was first used in the context of population genetics by R. A. Fisher (Fisher 1922; Fisher 1930) to study the survival of the progeny of a mutant gene and random fluctuations in the frequencies of genes. Subsequently, Haldane used the theory to investigate the problem of fixation of an advantageous allele (Haldane 1927).

Haldane demonstrated that the probability $\pi$, that a given genotype with selective advantage $s$ reaches fixation in a two-allele model, is given by the solution of the following equation (Haldane 1927):

$$
1-\pi=e^{-(1+s) \pi}
$$

For small selective values, the solution of this equation yields $\pi(s) \approx 2 s$. Recently, Barton (Barton 1995, Johnson and Barton 2002) extended the 


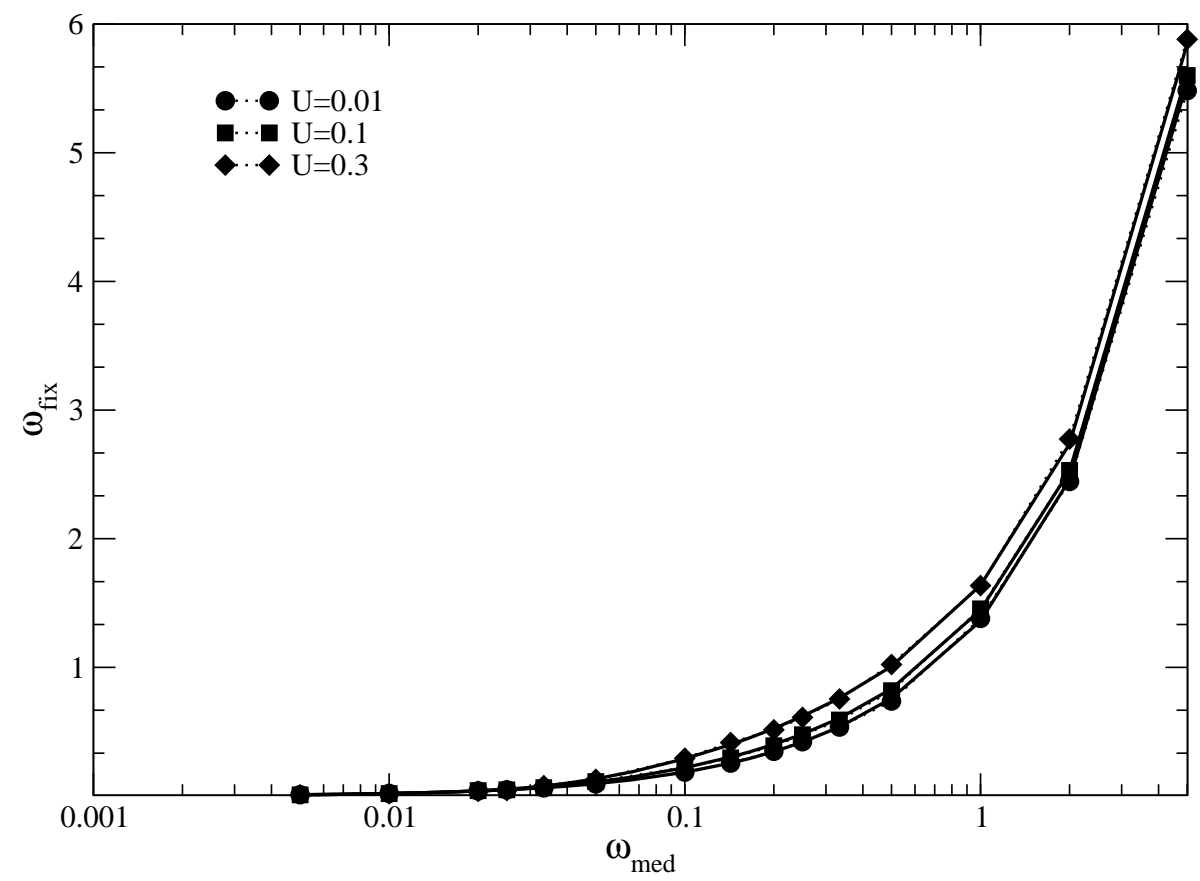

Figure 3: The mean value of the beneficial effect of the advantageous mutations that reached fixation $\omega_{f i x}$ as a function of $\omega_{\text {med }}$. The parameter values are $N=1000, s_{d}=0.1$ and from top to bottom $U=0.3, U=0.1$ and $U=0.01$. The data points correspond to the simulational data whereas the lines are the numerical solutions of Eq. (12).

use of the branching process formulation to heterogeneous genetic background, where the individuals can also produce offsprings which are not identical copies of itself. In the formulation, the probability $P_{i, t}$ that a beneficial mutation reaches fixation when it is present in a single genotype with genetic background $i$ (for instance, $i$ denotes the number of mutations) at generation $t$ is obtained by iterating the following set of equations:

$$
\left(1-P_{i, t-1}\right)=\sum_{j=0}^{\infty} W_{i, j}\left(1-P_{i, t}^{*}\right)^{j}
$$

where

$$
P_{i, t}^{*}=\sum_{k} M_{i, k} P_{k, t}
$$

is the probability that an allele in background $i$ at time $t-1$ would get fixed, 
given that at time $t$ it is passed to one offspring, and $M_{i, k}$ is an element of the mutation matrix that gives the chance that an offspring from a parent at background $i$ will be at background $k$. The quantity $W_{i, j}$ is the probability that an allele in background $i$ contributes with $j$ offsprings to the next generation. If the distribution of offsprings is given by a Poisson distribution with mean $\xi_{i}=w_{i} / \bar{w}$ (where $\bar{w}$ is the mean fitness population), then

$$
W_{i, j}=\frac{\xi_{i}^{j}}{j !} e^{-\xi_{i}}
$$

and Eq. (6) is written as

$$
\left(1-P_{i, t-1}\right)=\exp \left[-\xi_{i} P_{i, t}^{*}\right] .
$$

The probabilities of fixation correspond to the solution of Eq. (6) obtained in the limit $t \rightarrow \infty$, which we denote by $P_{i}=P_{i, t \rightarrow \infty}$.

We estimate the probability of fixation of a beneficial mutation with fixed selective effect $s_{b}, \Theta_{f i x}\left(s_{b}\right)$, by the sum over the distinct genetic backgrounds $i$

$$
\Theta_{f i x}=\sum_{i} P_{i} \bar{C}_{i}
$$

The concentrations of the classes $i, \bar{C}_{i}$, also means the chance of occurrence of the beneficial mutation in an individual in background $i$.

As we consider that the beneficial effect of an advantageous mutation is exponentially distributed according to Eq. (3), the probability of ultimate fixation $P_{f i x}$ of a beneficial mutation is then given by

$$
P_{f i x}=\int_{0}^{\infty} g\left(s_{b}\right) \Theta\left(s_{b}\right) d s_{b}
$$

Another relevant measurement in our statistics is the quantity $\omega_{\text {fix }}$, which represents the mean value of the beneficial effect of those mutations that have reached ultimate fixation, which we calculate as

$$
\omega_{f i x}=\frac{1}{P_{f i x}} \int_{0}^{\infty} s_{b} g\left(s_{b}\right) \Theta\left(s_{b}\right) d s_{b} .
$$

Although we only solve Equation (12) numerically, an analytical approximation can be obtained when we consider small values of the parameter $\omega_{\text {med }}=1 / \beta$ and zero mutation rate. In this case, the probability of fixation 


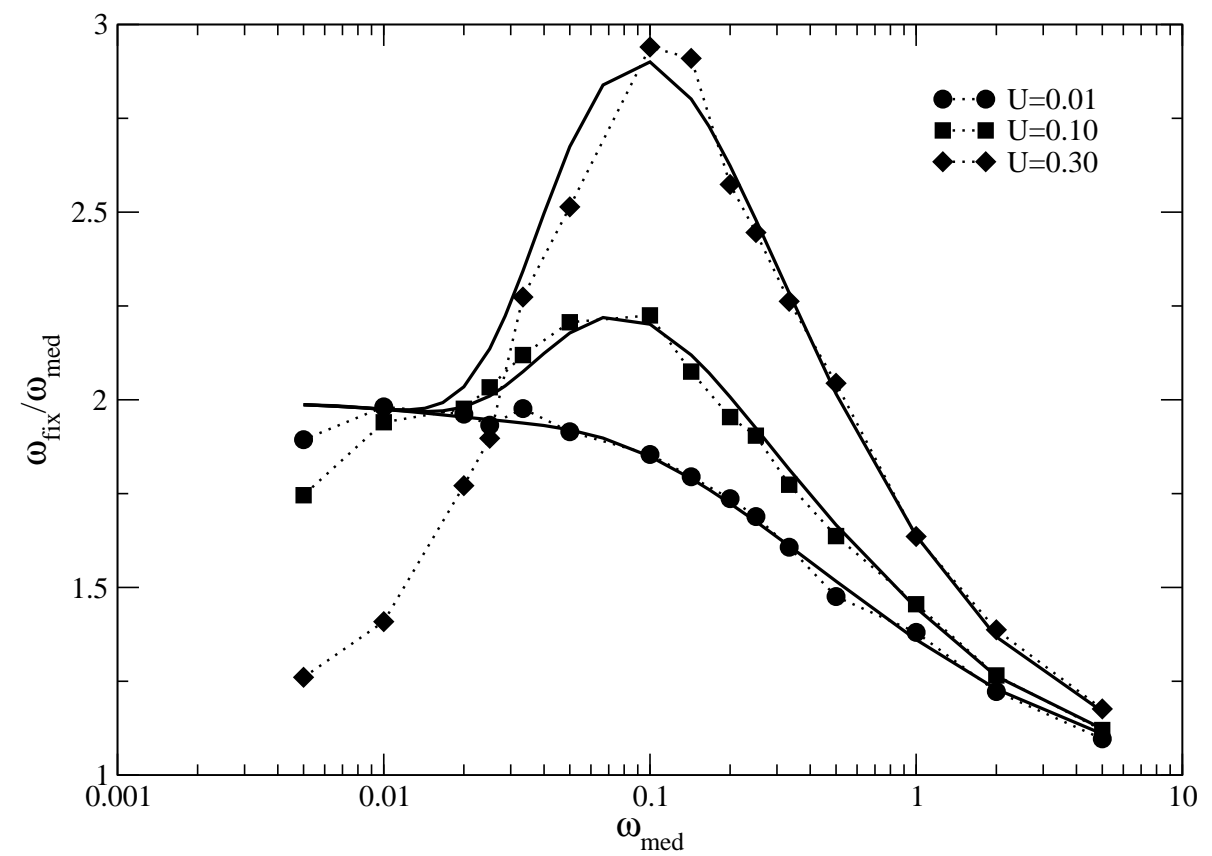

Figure 4: The ratio $\omega_{f i x} / \omega_{\text {med }}$ as a function of $\omega_{\text {med }}$. The parameter values are $N=1000, s_{d}=0.1$ and from top to bottom $U=0.3, U=0.1$ and $U=0.01$. The data points correspond to the simulational data whereas the lines are the numerical solutions of Eq. (12).

$\Theta\left(s_{b}\right)$ is equal to the solution for the two-allele model (see Eq. (57)), i.e., $\Theta\left(s_{b}\right) \approx 2 s_{b}$. Substituting the expression $\Theta\left(s_{b}\right)=2 s_{b}$ into Eqs. (11) and (12) we get

$$
\omega_{f i x}=\frac{2}{\beta},
$$

and the ratio between $\omega_{f i x}$ and $\omega_{m e d}$ yields the value 2 .

\section{Results and Discussions}

In this section we present and discuss our results from the simulations and also from the theoretical formulation.

In Figure 1 we plot the probability of ultimate fixation as a function of the mutation rate for parameter values $\beta=10$ and $s_{d}=0.1$. We obtain a good agreement between the simulational data and the theoretical curve obtained 
integrating numerically Eq. (11). The simulation results were taken over 100, 000 distinct runs. As expected, we observe a continous decrease of the probability $P_{f i x}$ as we increase the mutation value $U$. For high mutation rate $U$ the agreement between the simulations and the theoretical prediction is less satisfactory than those seen for small and intermediate values of $U$. This problem occurs due to the occurrence of the Muller's ratchet phenomenon in finite populations, at which the continuous accumulation of deleterious mutations leads to loss of the best adapted classes of individuals as the population evolves. Thus, for very high $U$ the population never reaches the equilibrium regime as supposed in the theoretical formulation (Campos 2003).

In Figure 2 we display the probability $P_{f i x}$ as a function of the mean value of the distribution of selective effects $\omega_{m e d}$ for fixed values of $U$. The probability $P_{f i x}$ is an increase function of the parameter $\omega_{m e d}$ since those mutations with higher selective effect have a greater chance of reaching fixation. We observe that this relation is well described by a power-law scaling, i.e., $P_{\text {fix }} \sim \omega_{\text {med }}^{\gamma}$ where the exponent $\gamma$ is an increase function of the mutation rate $U$, whereas $P_{\text {fix }}$ decreases with $U$, as we can see in Figure 1 .

Figure 3 shows the mean value of the selective advantages of those mutations that reached fixation $\omega_{\text {fix }}$ plotted against the mean value $\omega_{\text {med }}$. As expected $\omega_{f i x}$ increases with the augment of $\omega_{m e d}$, although we do not see a linear response. Besides $\omega_{f i x}$ increases with the raise of $U$, and in this case $U$ only those mutations awarding a large beneficial effect to the individuals have a non-negligible chance to reach fixation. We can better understand this scenario in Figure 4 where we plot the ratio $\omega_{f i x} / \omega_{m e d}$ as a function of $\omega_{\text {med }}$. From the figure, we see that the ratio $\omega_{f i x} / \omega_{\text {med }}$ is an increase function of the mutation rate $U$ for about all values of the parameter $\omega_{\text {med }}$. Nevertheless, an exception is obtained when we consider very small $\omega_{\text {med }}$, where we observe that when $U \rightarrow 0$ the ratio $\omega_{\text {fix }} / \omega_{\text {med }} \rightarrow 2$. Moreover, we witness that for intermediate to high values of $U$ the ratio $\omega_{f i x} / \omega_{\text {med }}$ is optimized around $\omega_{c} \approx 0.1$.

\section{Conclusions}

We have studied the dynamics of fixation of advantageous mutants in the multiplicative fitness landscape. We have investigated the problem by means of extensive simulations and also by a theoretical approach where we use the branching process formulation introduced by Haldane and extended by Bar- 
ton to the case of heterogeneous genetic background. The simulation results are in very good accordance with the theoretical predictions. The beneficial effect of these advantageous mutations is assumed to be exponentially distributed with mean value $\omega_{\text {med }}$. At once, we witness that the continuous supply of deleterious mutations to the population reduces the chance of fixation of the beneficial variants in the population. We have noticed that the relation between the probability of ultimate fixation $P_{f i x}$ and the parameter $\omega_{\text {med }}$ obeys a power-law scaling like $P_{\text {fix }} \sim \omega_{\text {med }}^{\gamma}$, where the exponent $\gamma$ depends on the mutation rate $U$. Surprisingly, we have observed that the ratio $\omega_{f i x} / \omega_{\text {med }}$ between the selective advantages of those mutations that reached fixation and the mean value of the distribution is optimized around the critical value $\omega_{\text {med }}=\omega_{c}=0.1$ for intermediate to high values of $U$. In this range of $U$, we have also witnessed that an increase of $U$ also means a higher value of $\omega_{\text {fix }} / \omega_{\text {med }}$. For very low values of $\omega_{\text {med }}$, the branching process theory fails to predict $\omega_{f i x} / \omega_{\text {med }}$ when we consider large values of $U$ : while the simulations show that the ratio $\omega_{\text {fix }} / \omega_{\text {med }}$ goes to 1 , which corresponds to a random stochastic regime, the theoretical analysis gives $\omega_{\text {fix }} / \omega_{\text {med }}=2$, which is the same value attained when we have mutation rate $U=0$. Actually, a smaller value of $U$ means that the random stochastic regime will be attained at smaller values of $\omega_{\text {med }}$.

\section{Acknowledgments}

VMO and PRAC are supported by Fundação de Amparo à Pesquisa do Estado de São Paulo under Proj. No. 03/00182-0. 


\section{References}

Barton, N. H. (1995). Linkage and the limits to natural selection. Genetics $140,821-841$.

Campos, P. R. A. (2003). Fixation of beneficial mutations in the presence of epistatic interactions. Bull. Math. Biol., in press.

Campos, P. R. A., C. Adami, and C. O. Wilke (2003). Modelling stochastic clonal interference. To appear in Modeling in Molecular Biology (Springer Series in Natural Computing).

Campos, P. R. A., M. T. Sonoda, and J. F. Fontanari (2000). On the structure of genealogical trees in the presence of selection. Physica A 283, $11-16$.

Colato, A. and J. F. Fontanari (2001). Soluble model for the accumulation of mutations in asexual populations. Phys. Rev. Lett. 87, art. no. 238102.

Cuevas, J. M., S. F. Elena, and A. Moya (2002). Molecular basis of adaptive convergence in experimental populations of rna viruses. Genetics 162, $533-542$.

de Visser, J. A. G. M., C. W. Zeyl, P. J. Gerrish, J. L. Blanchard, and R. E. Lenski (1999). Diminishing returns from mutation supply rate in asexual populations. Science 283, 404-406.

Fisher, R. A. (1922). On the dominance ratio. Proc. Roy. Soc. Edinb. Sect. B Biol. Sci. 42, 321-341.

Fisher, R. A. (1930). The Genetical Theory of Natural Selection. Claredon Press.

Gerrish, P. (2001). The rythm of microbial adaptation. Nature 413, 299302.

Gerrish, P. J. and R. E. Lenski (1998). The fate of competing beneficial mutations in an asexual population. Genetica 102, 127-144.

Gillespie, J. H. (1991). The Causes of Molecular Evolution. Oxford University Press.

Haldane, J. B. S. (1927). A mathematical theory of natural and artificial selection. part v: Selection and mutation. Proc. Camb. Phil. Soc. 26, 220-230. 
Harris, T. E. (1963). The Theory of Branching Processes. Springer.

Hill, W. G. and A. Robertson (1966). The effect of linkage on the limits to artificial selection. Genet. Res. 8, 269-294.

Johnson, T. and N. H. Barton (2002). The effect of deleterious alleles on adaptation in asexual organisms. Genetics 162, 395-411.

Kimura, M. and J. F. Crow (1964). The number of alleles that can be maintained in a finite populations. Genetics 49, 725-738.

Miralles, R., P. J. Gerrish, A. Moya, and S. F. Elena (1999). Clonal interference and the evolution of rna viruses. Science 285, 1745-1747.

Orr, H. A. (2000). The rate of adaptation in asexuals. Genetics 155, 961968.

Orr, H. A. (2003). The distribution of fitness effects among beneficial mutations. Genetics 163, 1519-1526.

Peck, J. R. (1994). A ruby in the rubbish: Beneficial mutations, deleterious mutations and the evolution of sex. Genetics 137, 597-606.

Rozen, D. E., J. A. G. M. de Visser, and P. J. Gerrish (2002). Fitness effects of fixed beneficial mutations in microbial populations. Curr. Biol. 12, 1040-1045.

Shaver, A. C., P. G. Dombrowski, J. Y. Sweeney, T. Treis, R. M. Zappala, and P. D. Sniegowski (2002). Fitness evolution and the rise of mutator alleles in experimental eschirichia coli populations. Genetics 162, 557566 .

Watterson, G. A. (1975). Number of segregating sites in genetic models without recombination. Theor. Popul. Biol. 10, 256-276.

Wilke, C. O. (2003). Probability of fixation of an advantageous mutant in a viral quasispecies. Genetics 162, 467-474. 